(c) 2021 Universidad Nacional Autónoma de México, Facultad de Estudios Superiores Zaragoza.

Este es un artículo Open Access bajo la licencia CC BY-NC-ND (http://creativecommons.org/licenses/by-nc-nd/4.0/).

TIP Revista Especializada en Ciencias Químico-Biológicas, 24: 1-7, 2021.

https://doi.org/10.22201/fesz.23958723e.2021.406

\title{
Almidón resistente como prebiótico y sus beneficios en el organismo humano
}

\author{
Victoria Montserrat Olayo-Contreras, San Juana Alemán-Castillo, \\ Guadalupe Rodríguez-Castillejos y Octelina Castillo-Ruiz* \\ Unidad Académica Multidisciplinaria Reynosa-Aztlán, Universidad \\ Autónoma de Tamaulipas, Calle 16 y Lago de Chapala s/n, Colonia Aztlán, \\ Reynosa 88740, Tamaulipas, México. E-mail: *ocastillo@uat.edu.mx
}

\section{RESUMEN}

Los almidones resistentes están formados por dos polímeros, amilosa y amilopectina con capacidad de resistir la digestión y mantenerse intactos en el intestino; se han relacionado con la prevención y control de enfermedades como la obesidad, la diabetes mellitus, las enfermedades cardiovasculares y el cáncer. De acuerdo con diversas investigaciones, se determinó que el almidón resistente cumple con las características necesarias para ser catalogado como prebiótico. El consumo adecuado de estas fibras que se encuentran en los cereales, las leguminosas y los tubérculos, ayudarían a reducir las comorbilidades provocadas por las enfermedades antes mencionadas. El objetivo de este trabajo de revisión fue recopilar información sobre los beneficios del almidón resistente y su relación con la salud.

Palabras clave: almidón, prebióticos, nutrición, microorganismos, salud.

\section{Resistant starch as prebiotic and its benefits in the human organism}

\begin{abstract}
Resistant starches are made up of two polymers, amylose and amylopectin that have the ability to resist digestion and remain intact in the intestine, they have been linked to the prevention and control of diseases including obesity, diabetes mellitus, cardiovascular disease and cancer. According to various investigations, it was determined that resistant starch meets the necessary characteristics to be catalogued a probiotic. The adequate consumption of these fibers that are found in cereals, legumes and tubers could help reduce the comorbidities caused by the diseases mentioned. The objective of this revision assignment is to identify information about the benefits of resistant starches and it relationship to health.
\end{abstract}

Key words: starch, prebiotics, nutrition, microorganisms, health. 


\section{INTRODUCCIÓN}

\section{Almidón resistente como prebiótico}

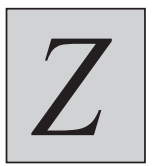
i-Ni, Rosma, Napisah, Karim \& Liong (2015), señalan que un producto es considerado un 'prebiótico' cuando presenta las siguientes características: resistente tanto a la acidez gástrica como a la hidrólisis provocadas por las enzimas y la absorción intestinal, la microflora intestinal lo fermente y, por último, con la capacidad de estimular el crecimiento y/o la actividad de las bacterias intestinales asociadas con la salud y el bienestar.

Jaiturong et al. (2020); Miketinas, Shankar, Maiya \& Patterson (2020), mencionan que el almidón resistente (AR) es benéfico para la salud al estimular el crecimiento y/o la actividad de cantidades limitadas de bacterias, así como descomponer las moléculas prebióticas por fermentación bacteriana en ácidos grasos de cadena corta (AGCC), específicamente acetato, propionato y butirato. Raigond, Ezekiel \& Raigond (2015) por su parte mencionan que el almidón resistente es prebiótico y simbiótico, ya que estimula la reproducción de bacterias probióticas e interactúa con otras fibras prebióticas como los $\beta$-glucanos.

La mayoría de las bacterias productoras de AGCC pertenecen a distintas familias como la Firmicutes que son bacterias productoras de butirato, esta misma función tiene las bacterias Spirochaete, Proteobacteria, Thermotogue, Fusobacteria y Actinobacteria, además de otras bacterias pertenecientes a Faecalibacterium, Roseburia y Eubacterium que producen butirato a partirdel acetatoy lactato(Peredo-Lovillo, Romero-Luna \& Jiménez-Fernández, 2020). Por otro lado, a las Bifidobacterias se les considera benéficas, ya que su influencia radica en la modulación del sistema inmunológico y el metabolismo, su predominio en el intestino ayuda a mejorar la peristalsis intestinal y reducir la fermentación de las bacterias en descomposición. El crecimiento de estos microorganismos se puede aumentar con el consumo de AR, especialmente el que se encuentra en las semillas de loto, soja verde y papa (Bao et al., 2017).

La comunidad de microorganismos que habitan en el tracto gastrointestinal concretamente las bacterias comensales modulan diversas funciones y son capaces de formar una barrera ante los patógenos, estimulando el sistema inmunológico en la supresión de señales inflamatorias por la producción de AGCC (Ghiamati, Soleimanian-Zad, van den Worm \& Folkerts, 2019).

De acuerdo con Markowiak \& Śliżewska (2017) la calidad alimentaria es uno de los aspectos fundamentales relacionados con la obesidad, las enfermedades cardiovasculares y el cáncer. Es por ello que diferentes investigaciones mencionan el uso de prebióticos como una alternativa nutricional que aporta beneficios a la salud humana. De acuerdo con Sanders, Merenstein, Reid, Gibson \& Rastall (2019) los prebióticos han servido para manipular el microbioma de sus hospedadores, por ello, Zaman \& Sarbini (2016) definen al prebiótico como un ingrediente alimentario no digerible que estimula el crecimiento de bacterias benéficas en el colon para mejorar la salud intestinal del consumidor.

Generalmente los prebióticos se conforman de polímeros de carbohidratos, que el intestino delgado no absorbe ni digiere y la mayoría se clasifican como fibras. Como parte de estas, se encuentran los 'almidones resistentes' (AR), que evitan como ya se mencionó los procesos de digestión en el intestino delgado hasta llegar al colon, donde finalmente son fermentados por microorganismos propios del microbioma del colon y producir ácidos grasos de cadena corta (AGCC) (DeMartino \& Cockburn, 2020).

Actualmente, los almidones resistentes como se expresó en líneas anteriores son por sus propiedades un medio que promete prevenir el riesgo de enfermedades crónicas como el cáncer colorrectal, la diabetes mellitus (tipo 1 y 2), la obesidad y las enfermedades cardiovasculares (Khan et al., 2019).

\begin{abstract}
Almidón resistente
Para poder comprender el proceso de degradación del AR, es importante mencionar que los almidones son inicialmente hidrolizados en la boca por acción de la amilasa salival y posteriormente por la enzima pancreática $\alpha$-amilasa; reduciéndolos a moléculas pequeñas de disacáridos y polisacáridos que posteriormente interactúan con enzimas ubicadas al borde del cepillo del intestino delgado. Sin embargo, el AR pasa por el intestino delgado sin verse afectado por su resistencia a la hidrólisis, resistencia que depende de las proporciones amilosa-amilopectina (Harris, 2019; Maier et al., 2017). Este tipo de almidón es fermentado por las bacterias que habitan en el intestino grueso, formando AGCC. Por lo tanto, el AR cumple la función prebiótica como alimento para los microorganismos antes mencionados (Keenan et al., 2015; De Paiva et al., 2020).
\end{abstract}

Según el grado y velocidad con el que son digeridos los almidones se clasifican como: almidón de rápida digestión (responsables de aumentar los niveles de glucosa en sangre después de su ingestión), almidón de lenta digestión (que se digieren por completo en el intestino delgado sólo que en menor proporción a los de rápida digestión) y almidón resistente (Ma \& Boye, 2018). A su vez el AR se divide en subgrupos desde el punto de vista nutricional como: Tipo 1 (AR1) o atrapado físicamente (que no se hidroliza por el efecto barrera de las paredes celulares de las proteínas de los alimentos como frijoles o granos parcialmente molidos). Tipo 2 (AR2) o gránulos de almidón resistente (almidón natural como el de la papa y el del plátano). Tipo 3 (AR3) o almidón retrógrado (hidrolizado por sus concentraciones de amilosa y su cristalización durante el enfriamiento y almacenamiento después de su gelatinización). Tipo 4 (AR4), un grupo de almidones que han sido modificados 
químicamente (Jiang, Du, Jiang, Wang \& Du, 2020). Por último, el Tipo 5 (AR5) es una formación por lípidos-amilosa y largas cadenas de carbono que son la causa de la resistencia a la digestión (Raigond et al., 2015; Fu, Wang, Tan \& Wang, 2021) (Tabla I), en la Figura 1, se muestran las estructuras de los diferentes tipos de almidón resistente (Xia, Zhu, Wang, Cui \& Yan, 2018).

\section{Acción del almidón resistente en la fermentación}

El acetato, propionato y butirato, son los tres principales productos de la fermentación del AR en el intestino: su producción da inicio en el colon proximal incluyendo el ascendente y transversal debido a las altas concentraciones de sustratos para la fermentación en el mismo. La concentración de estos ácidos grasos puede ser de 70 a $150 \mathrm{mM}$, absorbiéndose rápidamente $\mathrm{y}$ al menos el $10 \%$ se desechan por las heces. Diversos mecanismos permiten que estos metabolitos regulen el pH intestinal, el volumen de las células epiteliales, la absorción de cationes $\left(\mathrm{Na}^{+}\right)$y que además promuevan la absorción de otros nutrientes (Hu, Lin, Zheng \& Cheung, 2018).

Estos metabolitos proporcionan alrededor del 60 al $70 \%$ de las necesidades energéticas de las células epiteliales del colon, siendo específicamente el butirato el que suministra energía para los colonocitos, directamente en el crecimiento y diferenciación de estas células (Fu, Liu, Zhu, Mou \& Kong, 2019). El propionato contribuye a la gluconeogénesis en el hígado, mientras que el acetato es producido por acetogénesis reductora, por lo que alcanza las concentraciones más altas entre los AGCC en la luz intestinal (Louis \& Flint, 2017).

Algunos estudios han revelado el impacto que tienen el almidón resistente tipo 1, 3 y 5 en el metabolismo de los carbohidratos, con mayor enfoque en la glucosa e insulina (Lejk, Myśliwiec \& Myśliwiec, 2019). Hald et al. (2016) refieren que el AR tipo 2 aumenta la producción de butirato y modifica la composición de la microbiota al incrementar las cantidades de Bifidobacterium, Eubacterium rectale y Ruminococcus bromii; además que el AR tipo 3 contribuye a la proliferación de E. rectale y $R$. bromii, mientras que el AR tipo 4 influye en diversos aspectos funcionales del microbioma como la multiplicación de Bifidobacteria adolescentis y Parabacteroides distasonis. Este último es comúnmente utilizado en la industria alimentaria para dar un aspecto de suavidad y textura pulposa; además puede almacenarse a un $\mathrm{pH}$ bajo y a temperaturas altas; en la nutrición proporciona beneficios metabólicos, como la modulación de la resistencia a la insulina y la homeostasis de la glucosa (Yuan et al., 2018).
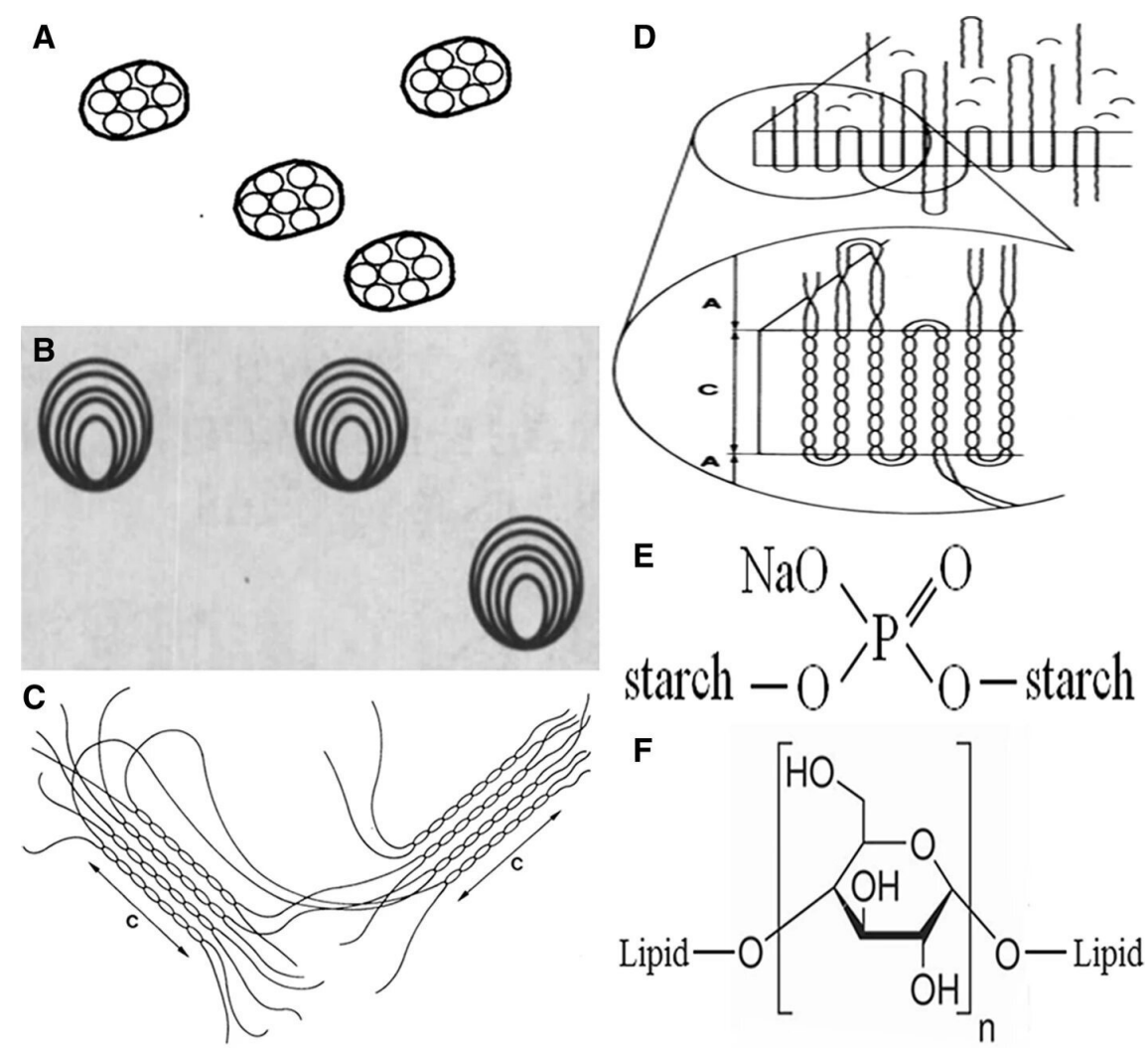

Figura 1. Estructura de almidón resistente: A) AR1, B) AR2, C) AR3: modelo de micelas, D) AR3: modelo de laminillas, E) AR4: almidón de fosforilación, F) AR5: complejos amilosa-lípidos. Fuente: Xia, Zhu, Wang, Cui \& Yan, 2018. 
Tabla I. Tipos de almidón resistente.

\begin{tabular}{|c|c|c|c|}
\hline $\begin{array}{l}\text { Tipos de } \\
\text { almidón }\end{array}$ & Alimentos en los que se encuentran & Características & Referencias \\
\hline AR1 & $\begin{array}{l}\text { Panes elaborados con granos enteros o molidos, } \\
\text { pastas elaboradas con trigo duro por extrusión, } \\
\text { frijoles }\end{array}$ & Atrapado físicamente & $\begin{array}{l}\text { Birt et al. (2013); Jiang et al. } \\
\text { (2020); Villarroel et al. (2018) }\end{array}$ \\
\hline AR2 & $\begin{array}{l}\text { Almidón de maíz con alto contenido de amilosa, } \\
\text { papa cruda, plátano verde, cebada, frijol, trigo, } \\
\text { guisantes, almidón de gingko }\end{array}$ & $\begin{array}{l}\text { Gránulos de almidón } \\
\text { resistente }\end{array}$ & $\begin{array}{l}\text { Birt et al. (2013); DeMartino \& } \\
\text { Cockburn (2020); Fu et al. (2021); } \\
\text { Lejk et al. (2019) }\end{array}$ \\
\hline AR3 & $\begin{array}{l}\text { Leguminosas, cereales (almidón de maíz, } \\
\text { hojuelas de maíz, pudines) y tubérculos (papas } \\
\text { crudas sin cocinar y cocidas, almidón de papa) }\end{array}$ & Almidón retrógrado & $\begin{array}{l}\text { Birt et al. (2013); DeMartino \& } \\
\text { Cockburn (2020); Jaiturong et al. } \\
\text { (2020); Keenan } \text { et al. (2015) }\end{array}$ \\
\hline AR4 & $\begin{array}{l}\text { No se encuentra de manera natural en los } \\
\text { alimentos, pero se puede agregar como } \\
\text { ingrediente funcional para mejorar el contenido } \\
\text { de fibra dietética en los alimentos, la harina } \\
\text { Fibersym }{ }^{\circledR} \text {, mejora la viscosidad y otras } \\
\text { características tecnológicas y sensoriales }\end{array}$ & Modificado químicamente & $\begin{array}{l}\text { Birt et al. (2013); DeMartino \& } \\
\text { Cockburn (2020); Patterson et al. } \\
\text { (2020); Villarroel et al. (2018); } \\
\text { Zaman \& Sarbini (2016) }\end{array}$ \\
\hline AR5 & $\begin{array}{l}\text { Procesamiento o cocción como el pan que } \\
\text { contiene grasa como ingrediente }\end{array}$ & Complejo lípido-amilosa & $\begin{array}{l}\text { DeMartino \& Cockburn (2020); } \\
\text { Lejk et al. (2019); Villarroel et al. } \\
\text { (2018) }\end{array}$ \\
\hline
\end{tabular}

\section{Beneficios para la salud}

La dieta es el principal factor que influye en la estructura de la comunidad microbiana en el colon (Crost et al., 2018). Ahmadi et al. (2019) opinan que la relación entre el microbioma intestinal y la salud humana se ha convertido en uno de los descubrimientos científicos más importantes, debido a la compleja composición del ecosistema microbiano en el intestino y su influencia en la salud del huésped, cabe hacer mención que la alteración del microbioma intestinal (disbiosis) puede resultar en un estado de enfermedad, ya sea por una respuesta inflamatoria excesiva o por un sistema inmunológico deficiente, que está asociada con enfermedades como obesidad y diabetes. Fu et al. (2021), consideran que la relación entre la obesidad y la disfunción de la microbiota intestinal puede ser modificada por medio de una intervención en la dieta, principalmente con cambios en la estructura y función de la misma, para evitar el desarrollo de la obesidad. Estudios con ratones han demostrado que cepas prebióticas, en la que están incluidos lactobacilos y bifidobacterias o mezclas de ellas, limitan la ganancia de peso corporal (Alard et al., 2016). En otro estudio realizado con ratones alimentados con $\mathrm{AR}$, se vio una disminución en la grasa corporal y una mejoría en la tolerancia a la glucosa, asociada a los niveles elevados de GLP-1 y PYY (Zhou et al., 2015).

El uso de almidones resistentes ha sido estudiado de una manera muy amplia y general, a partir de sus propiedades saludables y funcionales como ingrediente alimentario y su papel en la salud intestinal, potencialmente por su producción de butirato (Birt et al., 2013). Bendiks, Knudsen, Keenan \& Marco (2020) en una revisión sistemática y metanálisis de 13 estudios de casos y controles concluyeron que el consumo de AR2 en humanos mejora los niveles de glucosa e insulina en ayunas, así como la sensibilidad a la insulina en pacientes diabéticos y pacientes con sobrepeso, también indicaron que en otros catorce estudios y veinte ensayos encontraron que el consumo de AR redujo el colesterol sérico total y el colesterol de baja densidad (LDL).

Zhang et al. (2020) argumentan que en pacientes con diabetes mellitus tipo II, se demostró que el consumo de AR3 desempeñó un papel reductor en los niveles de glucosa en sangre, mejoraron los resultados de la tolerancia a la glucosa vía oral y se redujeron los niveles de colesterol y triglicéridos. Esto sugiere que también podrían disminuir las dislipidemias, la resistencia a la insulina y recuperar la sensibilidad de la misma. Trivieri et al. (2020) evaluaron los efectos del AR en el medio intestinal en estudios pre-clínicos de cáncer colorrectal, y demostraron la capacidad que tiene de aumentar las concentraciones de los AGCC, principalmente los niveles de butirato en los pacientes; en el caso de individuos sanos que fueron suplementados con AR en la dieta, se encontró una proliferación significativa de la mucosa del colon, protegiéndolo de aductos del DNA producidos en los tejidos del recto por el consumo de carne roja.

Patterson, Maiya \& Stewart (2020) muestran que el consumo de estos almidones reduce la inflamación, la oxidación de grasa posprandial, el colesterol sérico y las lipoproteínas de 
baja densidad. Además, se ha comprobado que el almidón resistente tipo 2 estimula la reproducción de $R$. bromii, E. rectale y Bifidobacterium; además con la intervención de las Proteobacterias se mejora la disbiosis.

$R$. bromii resulta ser la bacteria del colon más abundante y principal degradadora del AR; se ha observado que la fermentación de estas bacterias en el tracto gastrointestinal, tienen como objetivo proporcionar una fuente de energía a los colonocitos, cumpliendo su función como barrera intestinal para la prevención del cáncer colorrectal y el control de la inflamación intestinal (Crost et al., 2018).

Estudios en humanos y modelos in vitro asocian el consumo de fibras con una mayor diversidad bacteriana y en consecuencia sugieren que la combinación de diferentes consumos de fibras ayuda a regular el peso corporal (Abreu et al., 2021). Además, Villarroel, Gómez, Vera \& Torres (2018) mencionan que los efectos sobre la saciedad, durante el desayuno y el almuerzo, disminuyen la ingesta de energía después del consumo de $8 \mathrm{~g}$ de $\mathrm{AR}$, esta respuesta puede ser por la disminución en la velocidad del vaciamiento gástrico y al mantenimiento de glicemias más estables a través del tiempo.

\section{ConClusiones}

El almidón resistente se encuentra en diversos alimentos, ya sea de manera natural o adicionada. Estos son capaces de resistir la digestión y mantenerse intactos en el intestino delgado; proporcionan beneficios a la salud por prevenir y controlar enfermedades como la obesidad, la diabetes mellitus, las enfermedades cardiovasculares y el cáncer. Con las características que presenta para ser catalogado como prebiótico, el AR se convierte en indispensable para aumentar su consumo en la dieta diaria, con el fin de influir en la modificación positiva del microbioma intestinal del colon humano.

\section{Agradecimientos}

Al Consejo Nacional de Ciencia y Tecnología.

A la Universidad Autónoma de Tamaulipas.

A la Maestría en Ciencias y Tecnología de Alimentos.

\section{REFERENCIAS}

Abreu, A. T., Milke-García, M. P., Argüello-Arévalo, G. A., Calderón-de la Barca, A. M., Carmona-Sánchez, R. I., Consuelo-Sánchez, A., Coss-Adame, E., García-Cedillo, M. F., Hernández-Rosiles, V., Icaza-Chávez, M. E., Martínez-Medina, J. N., Morán-Ramos, S., Ochoa-Ortíz, E., Reyes-Apocada, M., Rivera-Flores, R. L., ZamarripaDorsey., M., Zarate-Mondragon, R. \& Vázquez-Frías, R. (2021). Fibra dietaria y microbiota, revisión narrativa de un grupo de expertos de la Asociación Mexicana de Gastroenterología. Revista de Gastroenterología de México,
86(3). DOI: 10.1016/j.rgmx.2021.02.004

Ahmadi, S., Nagpal, R., Wang, S., Gagliano, J., Kitzman, D. W., Soleimanian-Zad, S., Sheikh-Zeinoddin, M., Read, R. \& Yadav, H. (2019). Prebiotics from acorn and sago prevent high-fat-diet-induced insulin resistance via microbiome-gut-brain axis modulation. Journal of Nutritional Biochemistry, 67, 1-13. DOI: 10.1016/j. jnutbio.2019.01.011

Alard, J., Lehrter, V., Rhimi, M., Mangin, I., Peucelle, V., Abraham, A. L., Mariadassou, M., Maguin, E., WaligoraDupriet, A. J., Pot, B., Wolowczuk, I. \& Grangette, C. (2016). Beneficial metabolic effects of selected probiotics on diet-induced obesity and insulin resistance in mice are associated with improvement of dysbiotic gut microbiota. Environmental Microbiology, 18(5), 1484-1497. DOI: 10.1111/1462-2920.13181

Bao, C., Zeng, H., Zhang, Y., Zhang, L., Lu, X., Guo, Z., Miao, S. \& Zheng, B. (2017). Structural characteristics and prebiotic effects of Semen coicis resistant starches (type 3 ) prepared by different methods. International Journal of Biological Macromolecules, 105, 671-679. DOI: 10.1016/j. ijbiomac.2017.07.091

Bendiks, Z. A., Knudsen, K. E. B., Keenan, M. J. \& Marco, M. L. (2020). Conserved and variable responses of the gut microbiome to resistant starch type 2. Nutrition Research, 77, 12-28. DOI: 10.1016/j.nutres.2020.02.009

Birt, D. F., Boylston, T., Hendrich, S., Jane, J. L., Hollis, J., Li, L., McClelland, J., Moore, S., Phillips G.J., Rowling, M., Schalinske, K., Scott, M. P. \& Schalinske, K. (2013). Resistant starch: promise for improving human health. Advances in Nutrition, 4(6), 587-601. DOI: 10.3945/ an. 113.004325

Crost, E. H., Le Gall, G., Laverde-Gomez, J.A., Mukhopadhya, I., Flint, H. J. \& Juge, N. (2018). Mechanistic Insights Into the Cross-Feeding of Ruminococcus gnavus and Ruminococcus bromii on Host and Dietary Carbohydrates. Frontiers in Microbiology, 9, 2558. DOI: 10.3389/fmicb.2018.02558

De Paiva, B. R., Esgalhado, M., Borges, N. A., Kemp, J. A., Alves, G., Leite, P., Macedo, R., Cardozo, L., de Brito, J. S. \& Mafra, D. (2020). Resistant starch supplementation attenuates inflammation in hemodialysis patients: a pilot study. International Urology and Nephrology, 52(3), 549-555. DOI: 10.1007/s11255-020-02392-3

DeMartino, P. \& Cockburn, D. W. (2020). Resistant starch: impact on the gut microbiome and health. Current Opinion in Biotechnology, 61, 66-71. DOI: 10.1016/j. copbio.2019.10.008

Fu, J., Wang, Y., Tan, S. \& Wang, J. (2021). Effects of banana resistant starch on the biochemical indexes and intestinal flora of obese rats induced by a high-fat diet and their correlation analysis. Frontiers in Bioengineering and Biotechnology, 9, 1-14. DOI: 10.3389/fbioe.2021.575724.

Fu, X., Liu,Z.,Zhu, C., Mou, H.\& Kong, Q. (2019). Nondigestible carbohydrates, butyrate, and butyrate-producing bacteria. 
Critical Reviews in Food Science and Nutrition, 59, S130 S152. DOI: 10.1080/10408398.2018.1542587

Ghiamati Yazdi, F., Soleimanian-Zad, S., van den Worm, E. \& Folkerts, G. (2019). Turmeric Extract: Potential Use as a Prebiotic and Anti-Inflammatory Compound? Plant Foods for Human Nutrition, 74(3), 293-299. DOI: 10.1007/ s11130-019-00733-x

Hald, S., Schioldan, A. G., Moore, M. E., Dige, A., Lærke, H. N., Agnholt, J., Bach Knudsen, K. E., Hermansen, K., Marco, M. L., Gregersen, S. \& Dahlerup, J. F. (2016). Effects of Arabinoxylan and Resistant Starch on Intestinal Microbiota and Short-Chain Fatty Acids in Subjects with Metabolic Syndrome: A Randomised Crossover Study. PloS One, 11(7), e0159223. DOI: 10.1371/journal.pone.0159223.

Harris, K. F. (2019). An introductory review of resistant starch type 2 from high-amylose cereal grains and its effect on glucose and insulin homeostasis. Nutrition Reviews, 77(11), 748-764. DOI: 10.1093/nutrit/nuz040

Hu, J., Lin, S., Zheng, B. \& Cheung, P. (2018). Short-chain fatty acids in control of energy metabolism. Critical Reviews in Food Science and Nutrition, 58(8), 1243-1249. DOI: 10.1080/10408398.2016.1245650

Jaiturong, P., Laosirisathian, N., Sirithunyalug, B., Eitssayeam, S., Sirilun, S., Chaiyana, W. \& Sirithunyalug, J. (2020). Physicochemical and prebiotic properties of resistant starch from Musa sapientum Linn., ABB group, cv. Kluai Namwa Luang. Heliyon, 6(12), e05789. DOI: 10.1016/j. heliyon.2020.e05789.

Jiang, F., Du, C., Jiang, W., Wang, L. \& Du, S. K. (2020). The preparation, formation, fermentability, and applications of resistant starch. International Journal of Biological Macromolecules, 150, 1155-1161. DOI: 10.1016/j. ijbiomac.2019.10.124.

Keenan, M. J., Zhou, J., Hegsted, M., Pelkman, C., Durham, H. A., Coulon, D. B. \& Martin, R. J. (2015). Role of resistant starch in improving gut health, adiposity, and insulin resistance. Advances in Nutrition, 6(2), 198-205. DOI: 10.3945/an.114.007419

Khan, A., Rahman, U. U., Siddiqui, S., Irfan, M., Shah, A. A., Badshah, M., Hasan, F., \& Khan, S. (2019). Preparation and characterization of resistant starch type III from enzymatically hydrolyzed maize flour. Molecular Biology Reports, 46(4), 4565-4580. DOI: 10.1007/s11033-01904913-5

Lejk,A., Myśliwiec, M. \& Myśliwiec,A. (2019). Effect of eating resistant starch on the development of overweight, obesity, and disorders of carbohydrate metabolism in children. Pediatric Endocrinology, Diabetes, and Metabolism, 25(2), 81-84. DOI: 10.5114/pedm.2019.85818

Louis, P. \& Flint, H. J. (2017). Formation of propionate and butyrate by the human colonic microbiota. Environmental Microbiology, 19(1), 29-41. DOI: 10.1111/14622920.13589
Ma, Z. \& Boye, J. I. (2018). Research advances on structural characterization of resistant starch and its structurephysiological function relationship: A review. Critical Reviews in Food Science and Nutrition, 58(7), 1059-1083. DOI: 10.1080/10408398.2016.1230537

Maier, T. V., Lucio, M., Lee, L. H., VerBerkmoes, N. C., Brislawn, C. J., Bernhardt, J., Lamendella, R., McDermott, J. E., Bergeron, N., Heinzmann, S. S., Morton, J. T., Gonzalez, A., Ackermann, G., Knight, R., Riedel, K., Krauss, R. M., Schmitt-Kopplin, P. \& Jansson, J. K. (2017). Impact of dietary resistant starch on the human gut microbiome, metaproteome, and metabolome. MBio, 8(5), e01343-17. DOI: $10.1128 / \mathrm{mBio} .01343-17$

Markowiak, P. \& Śliżewska, K. (2017). Effects of Probiotics, Prebiotics, and Synbiotics on Human Health. Nutrients, 9(9), 1021. DOI: 10.3390/nu9091021

Miketinas, D. C., Shankar, K., Maiya, M. \& Patterson, M. A. (2020). Usual Dietary Intake of Resistant Starch in US Adults from NHANES 2015-2016. The Journal of Nutrition, 150(10), 2738-2747. DOI: 10.1093/jn/nxaa232

Patterson, M. A., Maiya, M. \& Stewart, M. L. (2020). Resistant Starch Content in Foods Commonly Consumed in the United States: A Narrative Review. Journal of the Academy of Nutrition and Dietetics, 120(2), 230-244. DOI: 10.1016/j. jand.2019.10.019

Peredo-Lovillo, A., Romero-Luna, H. E. \& Jiménez-Fernández, M. (2020). Health promoting microbial metabolites produced by gut microbiota after prebiotics metabolism. Food Research International, 136, 109473 DOI: 10.1016/j. foodres.2020.109473

Raigond, P., Ezekiel, R. \& Raigond, B. (2015). Resistant starch in food: a review. Journal of the science of food and agriculture, 95(10), 1968-1978. DOI: 10.1002/jsfa.6966

Sanders, M. E., Merenstein, D. J., Reid, G., Gibson, G. R. \& Rastall, R.A. (2019). Probiotics and prebiotics in intestinal health and disease: from biology to the clinic. Nature Reviews. Gastroenterology \& Hepatology, 16(10), 605-616. DOI: $10.1038 / \mathrm{s} 41575-019-0173-3$

Trivieri, N., Panebianco, C., Villani, A., Pracella, R., Latiano, T. P., Perri, F., Binda, E. \& Pazienza, V. (2020). High Levels of Prebiotic Resistant Starch in Diet Modulate a Specific Pattern ofmiRNAs Expression ProfileAssociated to a Better Overall Survival in Pancreatic Cancer. Biomolecules, 11(1), 26. DOI: 10.3390/biom11010026

Villarroel, P., Gómez, C., Vera, C. \& Torres, J. (2018). Resistant starch: Technological characteristics and physiological interests. Revista Chilena de Nutrición, 45(3), 271-278. DOI: $10.4067 / \mathrm{s} 0717-75182018000400271$

Xia, J., Zhu, D., Wang, R., Cui, Y. \& Yan, Y. (2018). Crop resistant starch and genetic improvement: a review of recent advances. Theoretical and Applied Genetics, 131(12), 2495-2511. DOI: 10.1007/s00122-018-3221-4.

Yuan, H. C., Meng, Y., Bai, H., Shen, D. Q., Wan, B. C. \& 
Chen, L. Y. (2018). Meta-analysis indicates that resistant starch lowers serum total cholesterol and low-density cholesterol. Nutrition Research, 54, 1-11. DOI: 10.1016/j. nutres.2018.02.008

Zaman, S. A. \& Sarbini, S. R. (2016). The potential of resistant starch as a prebiotic. Critical Reviews in Biotechnology, 36(3), 578-584. DOI: 10.3109/07388551.2014.993590

Zhang, C., Dong, L., Wu, J., Qiao, S., Xu, W., Ma, S., Zhao, B. \& Wang, X. (2020). Intervention of resistant starch 3 on type 2 diabetes mellitus and its mechanism based on urine metabonomics by liquid chromatography-tandem mass spectrometry. Biomedicine \& Pharmacotherapy, 128, 110350. DOI: 10.1016/j.biopha.2020.110350

Zhou, J., Martin, R. J., Raggio, A. M., Shen, L., McCutcheon, K. \& Keenan, M. J. (2015). The importance of GLP-1 and PYY in resistant starch's effect on body fat in mice. Molecular Nutrition \& Food Research, 59(5), 1000-1003. DOI: 10.1002/mnfr.201400904

Zi-Ni, T., Rosma, A., Napisah, H., Karim, A. A. \& Liong, M. T. (2015). Characteristics of Metroxylon sagu resistant starch type III as prebiotic substance. Journal of Food Science, 80(4), H875-H882. DOI: 10.1111/1750-3841.12817 